\title{
The class number one problem for the non-abelian normal CM-fields of degree 24 and 40
}

\author{
by \\ YounG-Ho PARK (Seoul)
}

1. Introduction. We fix an algebraic closure of $\mathbb{Q}$. Assume that all the number fields are subfields of the field $\mathbb{C}$ of complex numbers. We let $c$ denote complex conjugation and recall that if $\mathbf{N}$ is a normal CM-field, then $c$ is in the center $Z(\mathbf{G})$ of its Galois group $\mathbf{G}$.

There are 11 possible Galois groups for non-abelian normal CM-fields $\mathbf{N}$ of degree 24: $C_{2} \times \mathcal{A}_{4}, \mathrm{SL}_{2}\left(\mathbf{F}_{3}\right), C_{3} \times Q_{8}, Q_{24}, C_{3} \rtimes C_{8}, D_{24}, C_{2} \times Q_{12}$, $C_{2} \times D_{12}, C_{4} \times D_{6}, C_{3} \times D_{8}$, and $V_{24}=C_{3} \rtimes D_{8}$. For two of them, namely $C_{2} \times \mathcal{A}_{4}$ and $\mathrm{SL}_{2}\left(\mathbf{F}_{3}\right)$, whose 3 -Sylow subgroups are not normal, there are exactly 3 such CM-fields with class number one (see [LLO]). For the other 9 groups, $\mathbf{N}$ contains a normal octic CM-subfield $\mathbf{N}_{8}$ and the relative class number of $\mathbf{N}_{8}$ divides that of $\mathbf{N}$ (see [LOO, Thm. 5]). Hence, $\mathbf{N}$ with Galois group $C_{3} \times Q_{8}$ or $Q_{24}$ have even relative class numbers since the quaternion octic CM-fields $\mathbf{N}_{8}$ have even relative class numbers ([LO2]). Moreover, the relative class numbers of CM-fields $\mathbf{N}$ with Galois group $C_{3} \rtimes C_{8}$ are greater than one (see [Lou4]) and there is only one dihedral CM-field of degree 24 with class number one (see [Lef]). Therefore, it remains to deal with the following 5 groups: $C_{2} \times Q_{12}, C_{2} \times D_{12}, C_{3} \rtimes D_{8}, C_{4} \times D_{6}$, and $C_{3} \times D_{8}$. We will prove:

Theorem 1. (1) There is only one normal CM-field $\mathbf{N}$ with Galois group $C_{2} \times D_{12}$ and class number one: $\mathbf{N}=\mathbf{K}_{3} \mathbf{N}_{8}$ where $\mathbf{K}_{3}$ is the non-normal cubic field defined by the polynomial $x^{3}-6 x-2$ and $\mathbf{N}_{8}=\mathbb{Q}(\sqrt{-3}, \sqrt{-4}, \sqrt{-7})$. Notice that $d_{\mathbf{K}_{3}}=2^{2} \cdot 3^{3} \cdot 7, d_{\mathbf{N}_{8}}=2^{8} \cdot 3^{4} \cdot 7^{4}$, and $d_{\mathbf{N}}=2^{32} \cdot 3^{28} \cdot 7^{12}$.

2000 Mathematics Subject Classification: Primary 11R29, 11R21, 11Y35.

Key words and phrases: CM-field, relative class number, class field theory.

This work is supported in part by the Ministry of Information \& Communication of Korea ("Support Project of University Information Technology Research Center" supervised by IITA). 
(2) There is only one normal CM-field $\mathbf{N}$ with Galois group $C_{4} \times D_{6}$ and relative class number one: $\mathbf{K}=\mathbf{K}_{3} \mathbf{N}_{8}$ where $\mathbf{K}_{3}$ is the non-normal totally real cubic field defined by the polynomial $x^{3}-10 x-10$ and $\mathbf{N}_{8}$ $=\mathbb{Q}(\exp (2 \pi i / 5), \sqrt{13})$. This $\mathbf{N}$ has class number one. Notice that $d_{\mathbf{K}_{3}}=$ $2^{2} \cdot 5^{2} \cdot 13, d_{\mathbf{N}_{8}}=5^{6} \cdot 13^{4}$, and $d_{\mathbf{N}}=2^{16} \cdot 5^{22} \cdot 13^{12}$.

(3) There is only one normal CM-field $\mathbf{N}$ with Galois group $C_{3} \times D_{8}$ and relative class number one: $\mathbf{K}=\mathbf{N}_{3} \mathbf{N}_{8}$ where $\mathbf{N}_{3}$ is the real cyclic cubic field defined by the polynomial $x^{3}-x^{2}-4 x-1$ and

$$
\mathbf{N}_{8}=\mathbb{Q}(\sqrt{13}, \sqrt{17}, \sqrt{-(9+\sqrt{13}) / 2})
$$

is a dihedral octic CM-field. This $\mathbf{N}$ has class number one. Notice that $d_{\mathbf{N}_{3}}=$ $13^{2}, d_{\mathbf{N}_{8}}=13^{4} \cdot 17^{4}$, and $d_{\mathbf{N}}=13^{20} \cdot 17^{12}$.

(4) The relative class numbers of normal CM-fields $\mathbf{N}$ with Galois group $C_{2} \times Q_{12}$ or $C_{3} \rtimes D_{8}$ are greater than one.

Let $\mathbf{N}$ be a non-abelian normal CM-fields of degree $8 p$ with $p \geq 5$ a prime and let $\mathbf{G}=\operatorname{Gal}(\mathbf{N} / \mathbb{Q})$. Since the Sylow $p$-subgroup of $\mathbf{G}$ is normal $\mathbf{N}$ is a cyclic extension of degree $p$ of a normal octic CM-field $\mathbf{N}_{8}$, and the relative class number $h_{\mathbf{N}_{8}}^{-}$of $\mathbf{N}_{8}$ divides that of $\mathbf{N}$ (see [LOO, Thm. 5]). Let us consider the $\mathrm{CM}$-fields $\mathbf{N}$ of degree 40 . There are 11 non-abelian finite groups $\mathbf{G}$ of order 40: $C_{5} \rtimes C_{8}$ (2 groups), $C_{2} \times D_{20}, C_{2} \times Q_{20}, C_{4} \times D_{10}, C_{2} \times F_{5,4}$, $C_{5} \times D_{8}, D_{40}, V_{40}=C_{5} \rtimes D_{8}, C_{5} \times Q_{8}$, and $Q_{40}$. Moreover, since the center $Z(\mathbf{G})$ of each of these eleven $\mathbf{G}$ 's contains an element of order 2, nothing prevents each of these groups from being the Galois group for some non-abelian normal CM-field $\mathbf{N}$ of degree 40. If $\mathbf{G}=D_{40}$ then $h_{\mathbf{N}}^{-}>1$ (see [Lef]). If $\mathbf{G}=C_{5} \times Q_{8}$ or $Q_{40}$ then $h_{\mathbf{N}_{8}}^{-}$is even (see [LO1]), hence $h_{\mathbf{N}}^{-}$is even. Moreover, if $\mathbf{G}=C_{5} \stackrel{1}{\rtimes} C_{8}=\left\langle a, b: a^{5}=b^{8}=1, b^{-1} a b=a^{4}\right\rangle=\left\langle\sigma, \tau: \sigma^{20}=1\right.$, $\left.\tau^{2}=\sigma^{5}, \tau^{-1} \sigma \tau=\sigma^{9}\right\rangle$ or $C_{5} \stackrel{2}{\rtimes} C_{8}=\left\langle a, b: a^{5}=b^{8}=1, b^{-1} a b=a^{2}\right\rangle=$ $\left\langle\sigma, \tau: \sigma^{10}=1, \tau^{4}=\sigma^{5}, \tau^{-1} \sigma \tau=\sigma^{3}\right\rangle$ then analysis similar to that in the proof of [Lou4, Thm. 5] shows $h_{\mathbf{N}}^{-}>1$. Therefore, it remains to deal with the following 6 groups: $C_{2} \times Q_{20}, C_{2} \times D_{20}, C_{5} \rtimes D_{8}, C_{4} \times D_{10}, C_{5} \times D_{8}$, $C_{2} \times F_{5,4}=C_{2} \times\left\langle a, b: a^{5}=b^{4}=1, b^{-1} a b=a^{2}\right\rangle=\left\langle\sigma, \tau: \sigma^{10}=\tau^{4}=1\right.$, $\left.\tau^{-1} \sigma \tau=\sigma^{7}\right\rangle$. We will prove:

Theorem 2. There is only one normal CM-field $\mathbf{N}$ of degree 40 with class number one: $\mathbf{N}=\mathbf{K}_{5} \mathbf{N}_{8}$, where $\mathbf{K}_{5}$ is the non-normal totally real quintic field defined by the polynomial $x^{5}-10 x^{3}+20 x+10$ and $\mathbf{N}_{8}=$ $\mathbb{Q}(\exp (2 \pi i / 5), \sqrt{-7})$. Notice that $d_{\mathbf{K}_{5}}=2^{4} \cdot 5^{5} \cdot 7^{2}, d_{\mathbf{N}_{8}}=5^{6} \cdot 7^{4}$, and $d_{\mathbf{N}}=2^{32} \cdot 5^{46} \cdot 7^{20}$. Its Galois group $G(\mathbf{N} / \mathbb{Q})$ is $C_{2} \times F_{5,4}$.

2. Lattices of subfields. According to the foregoing, it is natural to closely investigate the non-abelian normal CM-fields $\mathbf{N}$ of degree $8 p, p \geq 3$ 
an odd prime, with Galois group $\mathbf{G}$ isomorphic to one of the following five groups:

$$
\begin{aligned}
& C_{2} \times Q_{4 p}=\left\langle\sigma, \tau, u: \sigma^{2 p}=\tau^{2}=1, u^{2}=\sigma^{p},\right. \\
& \left.u^{-1} \sigma u=\sigma^{2 p-1}, \sigma \tau=\tau \sigma, \tau u=u \tau\right\rangle, \\
& C_{2} \times D_{4 p}=\left\langle\sigma, \tau, u: \sigma^{2 p}=\tau^{2}=u^{2}=1,\right. \\
& \left.u^{-1} \sigma u=\sigma^{2 p-1}, \sigma \tau=\tau \sigma, \tau u=u \tau\right\rangle, \\
& C_{p} \rtimes D_{8}=\left\langle\sigma, \tau, u: \sigma^{2 p}=\tau^{2}=1, u^{2}=\tau,\right. \\
& \left.u^{-1} \sigma u=\sigma^{2 p-1}, \sigma \tau=\tau \sigma, \tau u=u \tau\right\rangle, \\
& C_{4} \times D_{2 p}=\left\langle\sigma, \tau: \sigma^{4 p}=\tau^{2}=1, \tau^{-1} \sigma \tau=\sigma^{2 p-1}\right\rangle, \\
& C_{p} \times D_{8}=\left\langle\sigma, \tau: \sigma^{4 p}=\tau^{2}=1, \tau^{-1} \sigma \tau=\sigma^{2 p+1}\right\rangle .
\end{aligned}
$$

Then we have

Table 1

\begin{tabular}{cccccc}
\hline $\mathbf{G}$ & $C_{2} \times Q_{4 p}$ & $C_{2} \times D_{4 p}$ & $C_{p} \rtimes D_{8}$ & $C_{4} \times D_{2 p}$ & $C_{p} \times D_{8}$ \\
\hline$Z(\mathbf{G})$ & $\left\langle\sigma^{p}, \tau\right\rangle$ & $\left\langle\sigma^{p}, \tau\right\rangle$ & $\langle\tau\rangle$ & $\left\langle\sigma^{p}\right\rangle$ & $\left\langle\sigma^{2}\right\rangle$ \\
$D(\mathbf{G})$ & $\left\langle\sigma^{2}\right\rangle$ & $\left\langle\sigma^{2}\right\rangle$ & $\left\langle\sigma^{2} \tau\right\rangle$ & $\left\langle\sigma^{4}\right\rangle$ & $\left\langle\sigma^{2 p}\right\rangle$ \\
$c$ & $\sigma^{p}$ or $\tau$ & $\tau$ & $\tau$ & $\sigma^{2 p}$ & $\sigma^{2 p}$ \\
\hline
\end{tabular}

where $Z(\mathbf{G}), D(\mathbf{G})$, and $c \in Z(\mathbf{G})$ denote the center of $\mathbf{G}$, the derived subgroup of $\mathbf{G}$ and the complex conjugation of $\mathbf{G}$, respectively.

Lattice I

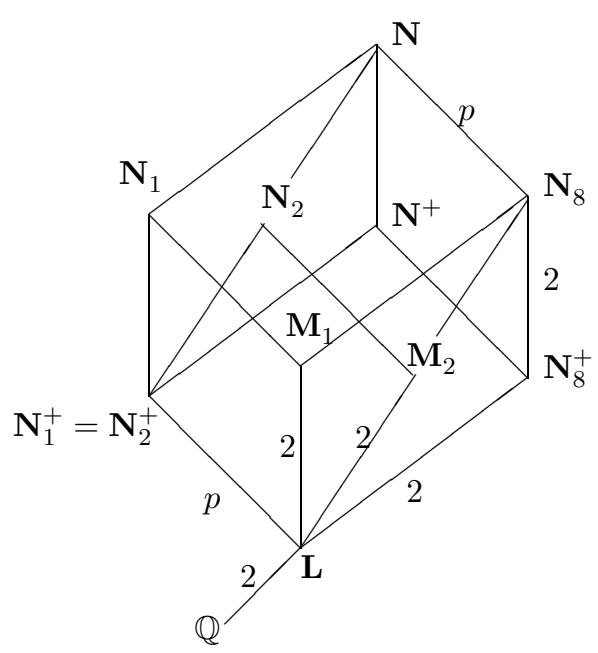

Lattice II

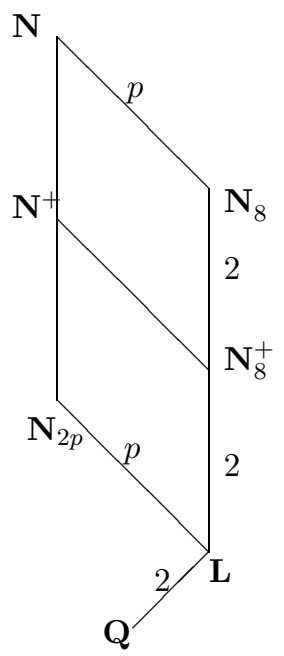

If $\mathbf{G}=C_{2} \times Q_{4 p}, C_{2} \times D_{4 p}$, or $C_{p} \rtimes D_{8}$, we let $\mathbf{L}, \mathbf{N}_{8}, \mathbf{N}_{1}$, and $\mathbf{N}_{2}$ be the fixed subfields of the subgroups $\langle\sigma, \tau\rangle,\left\langle\sigma^{2}\right\rangle,\left\langle\sigma^{p} \tau\right\rangle$ and $\left\langle c \sigma^{p} \tau\right\rangle$, respectively (Lattice I). Hence, $\mathbf{L}$ is a real quadratic subfield of $\mathbf{N}$, the extension $\mathbf{N} / \mathbf{L}$ is 
abelian with Galois group $G(\mathbf{N} / \mathbf{L}) \simeq C_{2 p} \times C_{2}$ and $\mathbf{N}_{8}$ is an octic CM-field containing $\mathbf{L}$. The field $\mathbf{N}$ is the compositum of two CM-fields $\mathbf{N}_{1}$ and $\mathbf{N}_{2}$ of degree $4 p$ with the same maximal real dihedral subfield $\mathbf{N}_{1}^{+}=\mathbf{N}_{2}^{+}$of degree $2 p$ containing $\mathbf{L}$. Let $\mathbf{M}_{i}=\mathbf{N}_{8} \cap \mathbf{N}_{i}$ for $i=1,2$. Then $\mathbf{N}_{8}$ is also the compositum of two CM-subfields $\mathbf{M}_{1}$ and $\mathbf{M}_{2}$ with the same maximal real quadratic subfield $\mathbf{L}$. We have

$$
h_{\mathbf{N}}^{-} / h_{\mathbf{N}_{8}}^{-}=\left(h_{\mathbf{N}_{1}}^{-} / h_{\mathbf{M}_{1}}^{-}\right)\left(h_{\mathbf{N}_{2}}^{-} / h_{\mathbf{M}_{2}}^{-}\right) .
$$

If $\mathbf{G}=C_{4} \times D_{2 p}$ or $C_{p} \times D_{8}$, we let $\mathbf{L}, \mathbf{N}_{8}$, and $\mathbf{N}_{2 p}$ be the fixed subfields of the cyclic subgroup generated by $\sigma, \sigma^{4}$, and $\sigma^{p}$, respectively (see Lattice II). Hence, the extension $\mathbf{N} / \mathbf{L}$ is cyclic. Considering $D(\mathbf{G})$ we can easily verify that $w_{\mathbf{N}}=w_{\mathbf{N}_{8}}$.

Table 2

Lattice I

\begin{tabular}{cccc}
\multicolumn{4}{c}{ Lattice I } \\
\hline$G(\mathbf{N} / \mathbb{Q})$ & $C_{2} \times Q_{4 p}$ & $C_{2} \times D_{4 p}$ & $C_{p} \rtimes D_{8}$ \\
\hline$G\left(\mathbf{N}_{8} / \mathbb{Q}\right)$ & $C_{4} \times C_{2}$ & $\left(C_{2}\right)^{3}$ & $D_{8}$ \\
\hline Remarks & $\mathbf{N}_{1}$ or $\mathbf{N}_{2}$ & $\mathbf{N}_{1}$ and $\mathbf{N}_{2}$ & $\mathbf{N}_{1} \simeq \mathbf{N}_{2}, \mathbf{M}_{1} \simeq \mathbf{M}_{2}$ \\
& dicyclic & dihedral & non-normal CM-fields \\
& & & $\mathbf{N}_{1} / \mathbf{L}$ cyclic \\
\hline
\end{tabular}

\begin{tabular}{ccc}
\multicolumn{3}{c}{ Lattice II } \\
\hline$G(\mathbf{N} / \mathbb{Q})$ & $C_{4} \times D_{2 p}$ & $C_{p} \times D_{8}$ \\
\hline$G\left(\mathbf{N}_{8} / \mathbb{Q}\right)$ & $C_{4} \times C_{2}$ & $D_{8}$ \\
\hline Remarks & $\mathbf{N}_{2 p}$ dihedral & $\mathbf{N}_{2 p}$ cyclic \\
& $\mathbf{N} / \mathbf{L}$ cyclic & $\mathbf{N} / \mathbf{L}$ cyclic \\
\hline
\end{tabular}

Let us set some notations we will use throughout this paper. If $\mathbf{N}$ is a number field, we let $d_{\mathbf{N}}, A_{\mathbf{N}}, w_{\mathbf{N}}, h_{\mathbf{N}}$, and $\zeta_{\mathbf{N}}$ denote the absolute value of its discriminant, its ring of integers, its number of complex roots of unity, its class number, and its Dedekind zeta function, respectively. If $\mathbf{N}$ is a CM-field, we let $\mathbf{N}^{+}, h_{\mathbf{N}}^{-}$and $Q_{\mathbf{N}} \in\{1,2\}$ denote its maximal real subfield, relative class number, and Hasse unit index, respectively (see [Wa]). If $\mathbf{L}$ is a quadratic number field, we let $\chi_{\mathbf{L}}$ denote the primitive quadratic Dirichlet character modulo $d_{\mathbf{L}}$ associated with $\mathbf{L}$. For any abelian extension $\mathbf{E} / \mathbf{F}$ let $\mathcal{F}_{\mathbf{E} / \mathbf{F}}$ be the finite part of its conductor and $f_{\mathbf{E} / \mathbf{F}}=N_{\mathbf{F} / \mathbb{Q}}\left(\mathcal{F}_{\mathbf{E} / \mathbf{F}}\right)$ the norm of the finite part of this conductor. Finally, we recall:

Proposition 3. (1) ([LOO, Th. 5]) Let $\mathbf{k} \subseteq \mathbf{K}$ be two CM-fields. Assume that $[\mathbf{K}: \mathbf{k}]$ is odd. Then $Q_{\mathbf{K}}=Q_{\mathbf{k}}$ and $h_{\mathbf{k}}^{-}$divides $h_{\mathbf{K}}^{-}$.

(2) ([LOO, Prop. 8]) Let $p$ be any odd prime number. Let $\mathbf{K} / \mathbf{M}$ be a cyclic extension of degree $p$ of $C M$-fields and let $\mathbf{K}^{+} / \mathbf{M}^{+}$also be cyclic. Let $t$ be the number of prime ideals of $\mathbf{M}^{+}$which split $\mathbf{M} / \mathbf{M}^{+}$and are ramified 
in $\mathbf{K}^{+} / \mathbf{M}^{+}$. Then $p^{t-1} h_{\mathbf{M}}^{-}$divides $h_{\mathbf{K}}^{-}$, and $p^{t} h_{\mathbf{M}}^{-}$divides $h_{\mathbf{K}}^{-}$if $p$ does not divide $w_{\mathbf{M}}$.

(3) ([LO1]) Let $t$ denote the number of prime ideals of $\mathbf{K}$ which are ramified in the quadratic extension $\mathbf{K} / \mathbf{K}^{+}$. Then $2^{t-1}$ divides $h_{\mathbf{K}}^{-}$.

(4) Let $p$ be an odd prime number. Let $\mathbf{K}$ be a real dihedral field of degree $2 p$ which is cyclic over a real quadratic field $\mathbf{L}$.

(a) ([LPL]) There exists a positive rational integer $F_{\mathbf{K} / \mathbf{L}}$ such that the conductor of $\mathbf{K} / \mathbf{L}$ is given by $\mathcal{F}_{\mathbf{K} / \mathbf{L}}=\left(F_{\mathbf{K} / \mathbf{L}}\right)$.

(b) ([Mar]) Let $\mathcal{Q}$ be a prime ideal of $\mathbf{L}$ above a rational prime $q$. If $q$ does not split in $\mathbf{L} / \mathbb{Q}$, then $\mathcal{Q}$ is not inert in $\mathbf{K} / \mathbf{L}$. Moreover, if $q$ is totally ramified in $\mathbf{K} / \mathbb{Q}$ then $q=p$. If $q \neq p$ and $\mathcal{Q}$ is ramified in $\mathbf{K} / \mathbf{L}$ then $q \equiv \chi_{\mathbf{L}}(q)(\bmod p)$.

2.1. Numerical computation of relative class numbers. We use the technique developed in [Lou5] and [Lou6] to compute efficiently relative class numbers of the CM-fields:

Proposition 4 (use [Lou6, Theorem 9]). Let $\mathbf{E}$ be a CM-field. Assume that there exists some totally real subfield $\mathbf{L}$ of $\mathbf{E}^{+}$such that the extension $\mathbf{E} / \mathbf{L}$ is cyclic of degree $2^{r} p$ with $r \geq 1$ and $p \geq 3$ any odd prime. Let $\mathbf{F}$ be a CM-subfield of $\mathbf{N}$ such that $\mathbf{L} \subseteq \mathbf{F} \subseteq \mathbf{E}$ and the degree of the extension $\mathbf{E} / \mathbf{F}$ is $p$. Finally, let $\chi$ be any one of the characters of order $2^{r} p$ associated with the cyclic extension $\mathbf{E} / \mathbf{L}$. Then $w_{\mathbf{E}} L(0, \chi)$ is an algebraic integer of the cyclotomic field $\mathbb{Q}\left(\zeta_{2^{r} p}\right), h_{\mathbf{F}}^{-}$divides $h_{\mathbf{E}}^{-}, w_{\mathbf{F}}$ divides $w_{\mathbf{E}}$, and

$$
h_{\mathbf{E}}^{-} / h_{\mathbf{F}}^{-}=\left(w_{\mathbf{E}} / w_{\mathbf{F}}\right) N_{\mathbb{Q}\left(\zeta_{2} r_{p}\right) / \mathbb{Q}}\left(\frac{1}{2^{m}} L(0, \chi)\right) .
$$

We refer the reader to [Lou6] to see how to use [Lou5] to compute the exact value of $L(0, \chi)$ (i.e. the values of the rational integers which are the coordinates in a given $\mathbb{Z}$-basis of the algebraic integer $w_{\mathbf{E}} L(0, \chi)$ ), prior to using (2).

TheOREM 5. Let $\mathbf{K}$ be a non-abelian normal $C M$-field of degree $2 n=2^{r} p$ $(r \geq 2)$ which is cyclic over a real quadratic field $\mathbf{L}$ and cyclic over a CMsubfield $\mathbf{K}_{2^{r}}$ of degree $2^{r}$. Assume also that $w_{\mathbf{K}}=w_{\mathbf{K}_{2^{r}}}$. Then $h_{\mathbf{K}_{2^{r}}}^{-}$divides $h_{\mathbf{K}}^{-}$and $h_{\mathbf{K}}^{-} / h_{\mathbf{K}_{2^{r}}}^{-}=\left(h_{\mathbf{K} / \mathbf{K}_{2^{r}}}^{-}\right)^{2}$ is a perfect square.

Proof. Let $\chi$ be any character of order $n$ associated with $\mathbf{K} / \mathbf{L}$. Then

$$
h_{\mathbf{K}}^{-} / h_{\mathbf{K}_{2^{r}}}^{-}=N_{\mathbb{Q}\left(\zeta_{n}\right) / \mathbb{Q}}\left(\frac{1}{4} L(0, \chi)\right)
$$

(Proposition 4). Let $\tau$ be the non-trivial element in $G(\mathbf{L} / \mathbb{Q})$. Then for any ideal $\mathcal{I}$ of $\mathbf{L}$ we have $\chi(\tau(\mathcal{I}))=\chi(\mathcal{I})^{k}$ where $k^{2} \equiv 1(\bmod n)$, which yields $\chi \circ \tau=\chi^{k}$. Let $\sigma_{k}$ denote the automorphism of $\mathbb{Q}\left(\zeta_{n}\right)$ which sends $\zeta_{n}$ to $\zeta_{n}^{k}$. Then $\sigma_{k}(L(0, \chi))=L\left(0, \chi^{k}\right)=L(0, \chi \circ \tau)=L(0, \chi)$ and $L(0, \chi)$ lies 
in the fixed subfield $\mathbf{F}$ of $\mathbb{Q}\left(\zeta_{n}\right)$ by $\sigma_{k}$. Therefore, we have $h_{\mathbf{K}}^{-} / h_{\mathbf{K}_{2^{r}}}^{-}=$ $N_{\mathbb{Q}\left(\zeta_{n}\right) / \mathbb{Q}}\left(\frac{1}{4} L(0, \chi)\right)=\left(N_{\mathbf{F} / \mathbb{Q}}\left(\frac{1}{4} L(0, \chi)\right)\right)^{2}$, which completes the proof.

\section{The case $\mathbf{G} \simeq C_{2} \times Q_{4 p}$}

Lemma 6 (due to $\mathrm{S}$. Louboutin). Let $\mathbf{N}_{1}$ and $\mathbf{N}_{2}$ be two distinct $C M$ fields with the same maximal totally real subfield. Set $\mathbf{N}=\mathbf{N}_{1} \mathbf{N}_{2}$. Assume that $Q_{\mathbf{N}_{1}}=1$ and that 4 does not divide $w_{\mathbf{N}_{1}}$. Then $h_{\mathbf{N}_{1}}^{-} h_{\mathbf{N}_{2}}^{-}$divides $2 h_{\mathbf{N}}^{-}$. In particular, if $G(\mathbf{N} / \mathbb{Q}) \simeq C_{2} \times Q_{4 p}$ then $h_{\mathbf{N}_{1}}^{-} h_{\mathbf{N}_{2}}^{-}$divides $2 h_{\mathbf{N}}^{-}$.

Proof. We have $h_{\mathbf{N}}^{-}=\eta_{\mathbf{N}} h_{\mathbf{N}_{1}}^{-} h_{\mathbf{N}_{2}}^{-}$, where $\eta_{\mathbf{N}}=Q_{\mathbf{N}} w_{\mathbf{N}} /\left(Q_{\mathbf{N}_{1}} w_{\mathbf{N}_{1}} Q_{\mathbf{N}_{2}} w_{\mathbf{N}_{2}}\right)$ $=Q_{\mathbf{N}} w_{\mathbf{N}} /\left(Q_{\mathbf{N}_{2}} w_{\mathbf{N}_{1}} w_{\mathbf{N}_{2}}\right)$ and $w_{\mathbf{N}_{1}} w_{\mathbf{N}_{2}}$ divides $2 w_{\mathbf{N}}$ (see [LO2, Proof of Prop. 2, point (b)]). We must prove that $2 \eta_{\mathbf{N}}$ is a positive rational integer. Clearly, we may assume that $w_{\mathbf{N}}=\frac{1}{2} w_{\mathbf{N}_{1}} w_{\mathbf{N}_{2}}$ and $Q_{\mathbf{N}_{2}}=2$. Now, we must prove that $Q_{\mathbf{N}}=2$. Since $Q_{\mathbf{N}_{2}}=2$, we have $W_{\mathbf{N}_{2}}=\left\langle\varepsilon_{2} / \bar{\varepsilon}_{2}\right\rangle$ for some $\varepsilon_{2} \in U_{\mathbf{N}_{2}}$, and since $Q_{\mathbf{N}_{1}}=1$ we have $W_{\mathbf{N}_{2}}^{2}=\left\langle\varepsilon_{1} / \bar{\varepsilon}_{1}\right\rangle$ for some $\varepsilon_{1} \in U_{\mathbf{N}_{1}}$. Now, $\phi:\left(\varepsilon, \varepsilon^{\prime}\right) \in W_{\mathbf{N}_{1}}^{2} \times W_{\mathbf{N}_{2}} \rightarrow \varepsilon \varepsilon^{\prime} \in W_{\mathbf{N}}$ is injective (for $W_{\mathbf{N}_{1}}^{2} \cap W_{\mathbf{N}_{2}} \subset$ $W_{\mathbf{N}_{1} \cap \mathbf{N}_{2}}=W_{\mathbf{N}_{1}^{+}}=\{ \pm 1\}$ and $\left.-1 \notin W_{\mathbf{N}_{1}}^{2}\right)$ and $\# \operatorname{Im} \phi=\frac{1}{2} w_{\mathbf{N}_{1}} w_{\mathbf{N}_{2}}=w_{\mathbf{N}}$. Thus, $\phi$ is surjective and $W_{\mathbf{N}} \subseteq U_{\mathbf{N}}^{1-c}$. Hence, $Q_{\mathbf{N}}=2$. If $G(\mathbf{N} / \mathbb{Q}) \simeq$ $Q_{4 p} \times C_{2}$, then we may assume that $\mathbf{N}_{1}$ is dicyclic. We have $Q_{\mathbf{N}_{1}}=Q_{\mathbf{M}_{1}}=1$ and $4 \nmid w_{\mathbf{N}_{1}}=w_{\mathbf{M}_{1}}$, where $\mathbf{M}_{1}$ is a cyclic quartic extension of $\mathbb{Q}$.

Proposition 7. Let $\mathbf{N}$ be a normal CM-field of degree $8 p$ with Galois group $G(\mathbf{N} / \mathbb{Q}) \simeq C_{2} \times Q_{4 p}$. If $p \equiv 3(\bmod 4)$, then $2^{p-2}$ divides $h_{\mathbf{N}}^{-}$which is therefore even. If $p \equiv 1(\bmod 4)$, then $h_{\mathbf{N}}^{-}>1$.

Proof. We may assume that $\mathbf{N}_{1}$ is a dicyclic CM-field of degree $4 p$. Then $2^{p-1}$ divides $h_{\mathbf{N}_{1}}^{-}$if $p \equiv 3(\bmod 4)($ see $[\mathrm{LOO}$, Thm. 6$])$ and $h_{\mathbf{N}_{1}}^{-} \geq 4$ in any case (see $[\mathrm{LP}])$. Therefore, using Lemma 6 , we get the desired results.

4. The case $\mathbf{G} \simeq C_{2} \times D_{4 p}$. Using the determination of all the dihedral CM-fields of degree $4 p$ with relative class number one and the determination of some imaginary abelian octic fields with class number one, we determine all the normal CM-fields $\mathbf{N}$ of degree $8 p$ with Galois group $G(\mathbf{N} / \mathbb{Q}) \simeq$ $C_{2} \times D_{4 p}$ which have class number one. We use the following lemma whose proof is left to the reader (use Hilbert class fields):

Lemma 8. Let $\mathbf{N}_{1}$ and $\mathbf{N}_{2}$ be two distinct CM-fields with the same maximal totally real subfield. Set $\mathbf{N}=\mathbf{N}_{1} \mathbf{N}_{2}$ and assume that $h_{\mathbf{N}}=1$. If neither $h_{\mathbf{N}_{1}}$ nor $h_{\mathbf{N}_{2}}$ is one, then $h_{\mathbf{N}_{1}}^{-}=h_{\mathbf{N}_{2}}^{-}=1$.

Theorem 9. There is only one normal CM-field of degree $8 p$ with Galois group $G(\mathbf{N} / \mathbb{Q}) \simeq C_{2} \times D_{4 p}$ of class number one: the one given in Theorem $1(1)$. 
Proof. Recall that $\mathbf{N}=\mathbf{N}_{1} \mathbf{N}_{2}$ is a compositum of two dihedral CMfields $\mathbf{N}_{1}$ and $\mathbf{N}_{2}$ of degree $4 p$ which are cyclic over the same real quadratic number field $\mathbf{L}$ and that $\mathbf{N}$ is a cyclic extension of degree $p$ of an elementary imaginary abelian octic field $\mathbf{N}_{8}$ containing $\mathbf{L}$ (see Lattice I and Table 2). We let $\mathcal{N}_{8}$ denote the finite set of all the elementary imaginary abelian octic fields $\mathbf{N}_{8}$ which are equal to their own genus field and have relative class number one. There are 17 such $\mathbf{N}_{8} \in \mathcal{N}_{8}$ : the 14 last fields in [Lou2, Table 2] and the following three: $\mathbb{Q}(\sqrt{-1}, \sqrt{-2}, \sqrt{-3}), \mathbb{Q}(\sqrt{-1}, \sqrt{-2}, \sqrt{-11})$ and $\mathbb{Q}(\sqrt{-1}, \sqrt{-2}, \sqrt{5})$ (see $[\mathrm{CK}]$ ). Since $h_{\mathbf{N}}=1$, the genus field of $\mathbf{N}_{8}$ is included in $\mathbf{N}$. Since $\mathbf{N}_{8}$ is the maximal abelian subfield of $\mathbf{N}, \mathbf{N}_{8}$ is its own genus field and $h_{\mathbf{N}_{8}}^{-}=1$ (use Proposition $3(1)$ ). Hence, $\mathbf{N}_{8} \in \mathcal{N}_{8}$. Moreover, according to Lemma 8 and noticing that no two of the 18 dihedral CM-fields of degree $4 p$ with relative class number one are cyclic extensions of the same real quadratic field (see [Lef, Th. 4.1]), we may assume that $\mathbf{N}_{1}$ is one of the 10 dihedral CM-fields of degree $4 p$ with class number one. Hence, $p=3$ or 5 . Now, only 5 out of these 10 dihedral CM-fields $\mathbf{N}_{1}$ are such that the imaginary biquadratic bicyclic subfield $\mathbf{M}_{1}$ of $\mathbf{N}_{1}$ is a subfield of one of the 17 fields $\mathbf{N}_{8} \in \mathcal{N}_{8}$. These five dihedral CM-fields are of degree 12 and are the ones of indices $i_{\mathbf{N}_{1}}=1,2,3,4$ and 6 in [Lef, Table 1, p. 85]. We have only the following 16 possible choices for the pair $\left(\mathbf{N}_{1}, \mathbf{N}_{8}\right)$ :

Table 3

\begin{tabular}{rrrrrrr}
\hline$i_{\mathbf{N}_{1}}$ & $\mathbf{M}_{1}$ & $d_{\mathbf{L}}$ & \multicolumn{1}{c}{$\mathbf{N}_{8}$} & \multicolumn{1}{c}{$\mathbf{M}_{2}$} & $h_{\mathbf{M}_{2}}^{-}$ & $h_{\mathbf{N}}^{-}$ \\
\hline 1 & $(-3,-15)$ & 5 & $(-3,-4,5)$ & $(-4,-20)$ & 1 & $3^{2}$ \\
1 & $(-3,-15)$ & 5 & $(-3,-7,5)$ & $(-7,-35)$ & 1 & $6^{2}$ \\
1 & $(-3,-15)$ & 5 & $(-3,-8,5)$ & $(-8,-40)$ & 1 & $3^{2}$ \\
\hline 2 & $(-3,-4)$ & 12 & $(-3,-4,5)$ & $(-15,-20)$ & 2 & $3^{2}$ \\
2 & $(-3,-4)$ & 12 & $(-3,-4,-7)$ & $(-7,-84)$ & 2 & $18^{2}$ \\
2 & $(-3,-4)$ & 12 & $(-3,-4,-11)$ & $(-11,-33)$ & 2 & $9^{2}$ \\
2 & $(-3,-4)$ & 12 & $(-3,-4,-19)$ & $(-19,-57)$ & 2 & $18^{2}$ \\
2 & $(-3,-4)$ & 12 & $(-1,-2,-3)$ & $(-24,-8)$ & 2 & $6^{2}$ \\
\hline 3 & $(-3,-7)$ & 21 & $(-3,-7,5)$ & $(-15,-35)$ & 2 & $3^{2}$ \\
$\mathbf{3}$ & $(-\mathbf{3},-\mathbf{7})$ & 21 & $(-\mathbf{3},-\mathbf{7},-\mathbf{4})$ & $(-\mathbf{4},-\mathbf{8 4})$ & $\mathbf{2}$ & $\mathbf{1}$ \\
3 & $(-3,-7)$ & 21 & $(-3,-7,-8)$ & $(-8,-168)$ & 2 & $3^{2}$ \\
\hline 4 & $(-3,-8)$ & 24 & $(-3,-8,5)$ & $(-15,-40)$ & 2 & $11^{2}$ \\
4 & $(-3,-8)$ & 24 & $(-3,-7,-8)$ & $(-7,-42)$ & 2 & $20^{2}$ \\
4 & $(-3,-8)$ & 24 & $(-1,-2,-3)$ & $(-4,-24)$ & 2 & $2^{2}$ \\
\hline 6 & $(-3,-19)$ & 57 & $(-3,-19,-4)$ & $(-4,-57)$ & 2 & $3^{2}$ \\
6 & $(-3,-19)$ & 57 & $(-3,-19,-11)$ & $(-11,-627)$ & 2 & $24^{2}$ \\
\hline
\end{tabular}

To compute $h_{\mathbf{N}}^{-}$, we use Theorem 5 . Note that according to (1) we have $h_{\mathbf{N}}^{-}=\left(h_{\mathbf{N}_{1}}^{-} / h_{\mathbf{M}_{1}}^{-}\right)\left(h_{\mathbf{N}_{2}}^{-} / h_{\mathbf{M}_{2}}^{-}\right)=h_{\mathbf{N}_{2}}^{-} / h_{\mathbf{M}_{2}}^{-}$. According to Table 3, Theorem 9 is proved. 
5. The cases $\mathbf{G} \simeq C_{p} \rtimes D_{8}, C_{4} \times D_{2 p}$ or $C_{p} \times D_{8}$. We use of the same plan as in $[\mathrm{LP}]$.

5.1. Lower bounds for relative class numbers

Theorem 10 (see the proof of [LP, Thm. 6]). Let $\mathbf{K}$ be a CM-field of degree $2 n$ which is cyclic of degree $2 m$ over an abelian real field $\mathbf{L}$. Let $f_{+}$denote the norm of the conductor of the extension $\mathbf{K}^{+} / \mathbf{L}$. Set $\varepsilon_{\mathbf{K}}=$ $\max \left\{\varepsilon_{\mathbf{K}}^{\prime}, \varepsilon_{\mathbf{K}}^{\prime \prime}\right\}$ where $\varepsilon_{\mathbf{K}}^{\prime}=\frac{2}{5} \exp \left(-2 \pi n / d_{\mathbf{K}}^{1 /(2 n)}\right), \varepsilon_{\mathbf{K}}^{\prime \prime}=1-\left(2 \pi n e^{1 / n} / d_{\mathbf{K}}^{1 /(2 n)}\right)$. Let $\mathbf{M} / \mathbf{L}$ be the only quadratic subextension of $\mathbf{K} / \mathbf{L}$. Assume that $\zeta_{\mathbf{M}}(s) \leq 0$ for $0<s<1$. Then for some constant $\mu_{\mathbf{L}}$ depending on $\mathbf{L}$ only, we have

$$
h_{\mathbf{K}}^{-} \geq \varepsilon_{\mathbf{K}} \frac{2 Q_{\mathbf{K}} w_{\mathbf{K}} \sqrt{d_{\mathbf{K}} / d_{\mathbf{K}^{+}}}}{e(2 \pi)^{n}\left(\operatorname{Res}_{s=1}\left(\zeta_{\mathbf{L}}\right)\right)^{m}\left(\frac{1}{2} \log f_{+}+2 \mu_{\mathbf{L}}\right)^{m-1} \log d_{\mathbf{K}}} .
$$

To compute the numerical approximations of $\operatorname{Res}_{s=1}\left(\zeta_{\mathbf{L}}\right)$ and $\mu_{\mathbf{L}}$ we use [Lou3].

TheOREM 11. (1) ([CK]) Let $\mathbf{N}_{8}=\mathbf{L}_{4} \mathbf{L}_{2}$ be an imaginary abelian octic field with Galois group $G\left(\mathbf{N}_{8} / \mathbb{Q}\right) \simeq C_{4} \times C_{2}$, where $\mathbf{L}_{4}$ is an imaginary cyclic quartic of conductor $f_{4}$ and $\mathbf{L}_{2}$ is a real quadratic of conductor $f_{2}$. Then $h_{\mathbf{N}_{8}}^{-}=1$ if and only if $\left(f_{4}, f_{2}\right) \in\{(5,8),(5,13),(5,17),(13,5),(13,8)$, $(16,5)\}$. For these six fields $\mathbf{N}_{8}$ we have $\zeta_{\mathbf{N}_{8}^{+}}(s) \leq 0$ for $0<s<1$.

(2) ([LO1]) There are exactly 19 dihedral octic $C M$-fields $\mathbf{N}_{8}$ with relative class number one: the narrow Hilbert 2-class fields of the 19 real quadratic field $\mathbf{L}$ which appear in Table 6. For these 19 fields $\mathbf{N}_{8}$ we have $\zeta_{\mathbf{N}_{8}^{+}}(s) \leq 0$ for $0<s<1$.

(3) ([LO1]) There are 38 non-normal quartic CM-fields $\mathbf{M}$ with relative class number one (they are pairwise isomorphic and their normal closures are the previous 19 dihedral octic CM-fields with relative class number one). For these 38 fields $\mathbf{M}$ we have $\zeta_{\mathbf{M}}(s) \leq 0$ for $0<s<1$.

COROllary 12. Let $p \geq 3$ be a given odd prime. We can compute an explicit bound on the discriminants of the normal CM-fields of degree $8 p$ with Galois groups $\mathbf{G} \simeq C_{p} \rtimes D_{8}, C_{4} \times D_{2 p}$, or $C_{p} \times D_{8}$ and relative class number one. More precisely:

(1) Assume that $\mathbf{G} \simeq C_{4} \times D_{2 p}$ or $C_{p} \times D_{8}$ (Lattice II). Then $h_{\mathbf{N}_{8}}^{-}=1$. For each of these $25(=6+19)$ CM-fields $\mathbf{N}_{8}$ of relative class number one we can compute a bound $B_{p}(\mathbf{L})$ on the norm $f_{+}$of the conductor of the extension $\mathbf{N}^{+} / \mathbf{L}$ such that $h_{\mathbf{N}}^{-}=1$ implies $f_{+} \leq B_{p}(\mathbf{L})$.

(2) Assume that $\mathbf{G} \simeq C_{p} \rtimes D_{8}$ (Lattice I). Then $h_{\mathbf{N}}^{-}=1$ if and only if $h_{\mathbf{N}_{1}}^{-}=1$. Now, $h_{\mathbf{N}_{1}}^{-}=1$ implies $h_{\mathbf{M}_{1}}^{-}=1$. For each of these 38 non-normal quartic CM-fields $\mathbf{M}_{1}$ of relative class number one we can compute a bound $B_{p}(\mathbf{L})$ on the norm $f_{+}$of the conductor of the extension $\mathbf{N}_{1}^{+} / \mathbf{L}$ such that $h_{\mathbf{N}_{1}}^{-}=1$ implies $f_{+} \leq B_{p}(\mathbf{L})$. 
Proof. First, assume that $\mathbf{G} \simeq C_{4} \times D_{2 p}$ or $C_{p} \times D_{8}$ (Lattice II). Then $\mathbf{N} / \mathbf{L}$ is a cyclic extension of degree $4 p$. For $s$ real we have

$$
\left(\zeta_{\mathbf{N}} / \zeta_{\mathbf{N}_{8}^{+}}\right)(s)=\prod_{\{\chi, \bar{\chi}\}, \chi^{2} \neq 1}|L(s, \chi)|^{2} \geq 0
$$

(where $\chi$ ranges over the $4 p-2$ non-quadratic characters associated with the extension $\mathbf{N} / \mathbf{L}$ ), and we conclude that $\zeta_{\mathbf{N}_{8}^{+}}(s) \leq 0$ for $0<s<1$ implies $\zeta_{\mathbf{N}}(s) \leq 0$ for $0<s<1$. Now, assume that $h_{\mathbf{N}}^{-}=1$. Since $h_{\mathbf{N}_{8}}^{-}$divides $h_{\mathbf{N}}^{-}$(Proposition 3), we have $h_{\mathbf{N}_{8}}^{-}=1$ and $\zeta_{\mathbf{N}_{8}^{+}}(s) \leq 0$ for $0<s<1$ (Theorem 11(1), (2)). On applying Theorem 10 with $\mathbf{K}=\mathbf{N}$ and $2 m=4 p$, for each of the finitely many $\mathbf{N}_{8}$ with $h_{\mathbf{N}_{8}}^{-}=1$ we obtain a good lower bound of $B_{p}(\mathbf{L})$. (See Tables 4,6 , and 7.)

Second, assume that $\mathbf{G} \simeq C_{p} \rtimes D_{8}$ (Lattice I). Then $\mathbf{M}_{1}$ is a nonnormal quartic CM-field, hence $Q_{\mathbf{M}_{1}}=1$ (see [Lou1, Lemma 1]), $Q_{\mathbf{N}_{1}}=$ $Q_{\mathbf{M}_{1}}=1$, by Proposition 3(1), and $h_{\mathbf{N}}^{-}=\left(Q_{\mathbf{N}} / 2\right)\left(h_{\mathbf{N}_{1}}^{-} / Q_{\mathbf{N}_{1}}\right)^{2}$ (see [LO2, Prop. 2]). Hence, $h_{\mathbf{N}}^{-}=1$ implies $h_{\mathbf{N}_{1}}^{-}=1$ (and $Q_{\mathbf{N}}=2$ ) and $h_{\mathbf{M}_{1}}^{-}=1$ (Proposition 3(1)). Conversely, $h_{\mathbf{N}_{1}}^{-}=1$ implies $h_{\mathbf{N}}^{-}=1$ (and $Q_{\mathbf{N}}=2$ ). Since $\mathbf{N}_{1} / \mathbf{L}$ is cyclic of degree $2 p$, as for the previous point we also obtain a good lower bound of $B_{p}(\mathbf{L})$. (See Table 9 .)

If we followed the same line of reasoning as in [Lef], we could determine all the normal CM-fields of degree $8 p, p \geq 3$ any odd prime, with Galois group $\mathbf{G} \simeq C_{p} \rtimes D_{8}, C_{4} \times D_{2 p}$ or $C_{p} \times D_{8}$ with relative class number one. Instead of determining all the normal CM-fields of degree $8 p$ we determine the fields of degree 24 and 40 in the following three sections. We will prove:

TheOREM 13. The only normal CM-fields of degree 24 with Galois group $G(\mathbf{N} / \mathbb{Q}) \simeq C_{3} \rtimes D_{8}, C_{4} \times D_{6}$, or $C_{3} \times D_{8}$ of relative class number one are the two fields given in Theorem 1(2), (3). If $\mathbf{N}$ is a normal CM-field of degree 40 with Galois group $G(\mathbf{N} / \mathbb{Q}) \simeq C_{5} \rtimes D_{8}, C_{4} \times D_{10}$, or $C_{5} \times D_{8}$, then $h_{\mathbf{N}}^{-}>1$.

5.2. The cases $\mathbf{G} \simeq C_{4} \times D_{6}$ and $\mathbf{G} \simeq C_{4} \times D_{10}$ (Lattice $\left.I I\right)$

Proposition 14. Let $\mathbf{N}$ be a normal CM-field of degree $8 p$ with $G(\mathbf{N} / \mathbb{Q})$ $\simeq C_{4} \times D_{2 p}$. Assume that $h_{\mathbf{N}}^{-}$is odd. If a rational prime $q$ is ramified in $\mathbf{N}_{8} / \mathbf{L}$, then $q$ divides $f_{\mathbf{N}_{2 p} / \mathbf{L}}$.

Proof. Suppose $q$ splits in $\mathbf{L} / \mathbb{Q}$. Then $t_{\mathbf{N}_{8} / \mathbf{N}_{8}^{+}} \geq 2$ and $2\left|h_{\mathbf{N}_{8}}^{-}\right| h_{\mathbf{N}}^{-}$by Proposition 3(1), (3), which is a contradiction. Hence, $q$ does not split in $\mathbf{L} / \mathbb{Q}$. Let $\mathcal{Q}_{\mathbf{L}}$ denote the prime ideal of $\mathbf{L}$ above $q$. According to Proposition $3(4), \mathcal{Q}_{\mathbf{L}}$ is not inert in $\mathbf{N}_{2 p} / \mathbf{L}$. Suppose $\mathcal{Q}_{\mathbf{L}}$ were not ramified in $\mathbf{N}_{2 p} / \mathbf{L}$. Then $\mathcal{Q}_{\mathbf{L}}$ would split in $\mathbf{N}_{2 p} / \mathbf{L}$. Since $\mathcal{Q}_{\mathbf{L}}$ is ramified in $\mathbf{N}_{8} / \mathbf{L}$ and since $\mathbf{N} / \mathbf{L}$ is cyclic, the $p$ prime ideals $\mathcal{Q}_{1}, \ldots, \mathcal{Q}_{p}$ of $\mathbf{N}_{2 p}$ above $\mathcal{Q}_{\mathbf{L}}$ would be ramified in the cyclic quartic extension $\mathbf{N} / \mathbf{N}_{2 p}$, hence the prime ideals 
of $\mathbf{N}^{+}$above $\mathcal{Q}_{i}$ would be ramified in the quadratic extension $\mathbf{N} / \mathbf{N}^{+}$, we would have $t_{\mathbf{N} / \mathbf{N}^{+}} \geq p$ and $2^{p-1}$ would divide $h_{\mathbf{N}}^{-}$(by Proposition $3(2)$ ). A contradiction. Hence, $\mathcal{Q}_{\mathbf{L}}$ is ramified in $\mathbf{N}_{2 p} / \mathbf{L}$ and $q$ divides $f_{\mathbf{N}_{2 p} / \mathbf{L}}$.

First, assume that $G(\mathbf{N} / \mathbb{Q}) \simeq C_{4} \times D_{6}$ and $h_{\mathbf{N}}^{-}=1$. Then $h_{\mathbf{N}_{8}}^{-}=1$ and $\mathbf{N}_{8}$ is known (Theorem 11(1)). In fact, we can get rid of two of the six possible fields $\mathbf{N}_{8}$ and we can decide which one of the three real quadratic subfields of a given $\mathbf{N}_{8}$ must be equal to $\mathbf{L}$ :

Lemma 15. If $h_{\mathbf{N}}^{-}=1$ then $\left(f_{4}, f_{2}, d_{\mathbf{L}}\right) \in\{(16,5,5),(5,8,8),(5,13,13)$, $(5,17,17)\}$.

Proof. If a rational prime $q$ divides $f_{\mathbf{N}_{8} / \mathbf{L}}$, then $q \in\{2,5,13\}$ and $q \mid f_{\mathbf{N}_{6} / \mathbf{L}}$ by Proposition 14. Note that if $q$ is ramified in $\mathbf{L} / \mathbb{Q}$, then $q$ is totally ramified in $\mathbf{N}_{6} / \mathbb{Q}$ and $q=3$. This implies that $q$ is inert in $\mathbf{L} / \mathbb{Q}$ and $3 \mid(q+1)$ by Proposition 3(4), which yields the desired result.

For the four fields $\mathbf{N}_{8}$ we compute $B_{3}(\mathbf{L})$ such that $h_{\mathbf{N}}^{-}>1$ if $f_{\mathbf{N}_{6} / \mathbf{L}}>$ $B_{3}(\mathbf{L})$. Let $n_{f}$ be the number of conductors of $\mathbf{N} / \mathbf{L}$ satisfying $f_{\mathbf{N}_{6} / \mathbf{L}} \leq$ $B_{3}(\mathbf{L})$ and $N_{f}$ the number of conductors of $\mathbf{N} / \mathbf{L}$ satisfying $f_{\mathbf{N}_{6} / \mathbf{L}} \leq B_{3}(\mathbf{L})$ and Proposition 14. We refer the reader to Table 4 for the result of our computation. Notice that $N_{f}$ is much small than $n_{f}$, which clearly shows how useful Proposition 14 is for alleviating the amount of computation required. Finally, in Table 5 we give the results of our relative class number computations. According to Table 5, there is only one such CM-field with $h_{\mathbf{N}}^{-}=1$. Notice that there are two fields $\mathbf{N}_{6}$ for which $\mathcal{F}_{\mathbf{N}_{6} / \mathbf{L}}=(5 \cdot 22)$. In the same way, for the case $G(\mathbf{N} / \mathbb{Q}) \simeq C_{4} \times D_{10}$ we computed Table 6 according to which there is no such $\mathbf{N}$ with $h_{\mathbf{N}}^{-}=1$.

Table $4\left(G(\mathbf{N} / \mathbb{Q}) \simeq C_{4} \times D_{6}\right)$

\begin{tabular}{rrrrcccrc}
\hline & $d_{\mathbf{L}}$ & $h_{\mathbf{L}}$ & $\mathrm{R}_{\mathbf{L}} \leq$ & $\mu_{\mathbf{L}} \leq$ & $f_{\mathbf{N}_{8} / \mathbf{L}}$ & $B_{3}(\mathbf{L})$ & $n_{f}$ & $N_{f}$ \\
\hline 1 & 5 & 1 & 0.431 & 0.1014 & $2^{8}$ & $30^{2}$ & 1 & 1 \\
2 & 8 & 1 & 0.624 & 0.1409 & $5^{2}$ & $310^{2}$ & 20 & 5 \\
3 & 13 & 1 & 0.663 & 0.2215 & $5^{2}$ & $230^{2}$ & 21 & 5 \\
4 & 17 & 1 & 1.017 & 0.2167 & $5^{2}$ & $390^{2}$ & 27 & 9 \\
\hline
\end{tabular}

Table $5\left(G(\mathbf{N} / \mathbb{Q}) \simeq C_{4} \times D_{6}\right)$

\begin{tabular}{rrcr|rrrr|rrrr}
\hline & $d_{\mathbf{L}}$ & $\mathcal{F}_{\mathbf{N}_{6} / \mathbf{L}}$ & $h_{\mathbf{N}}^{-}$ & & $d_{\mathbf{L}}$ & $\mathcal{F}_{\mathbf{N}_{6} / \mathbf{L}}$ & $h_{\mathbf{N}}^{-}$ & & $d_{\mathbf{L}}$ & $\mathcal{F}_{\mathbf{N}_{6} / \mathbf{L}}$ & $h_{\mathbf{N}}^{-}$ \\
\hline 1 & 5 & $\left(2 \cdot 3^{2}\right)$ & $65^{2}$ & 8 & 13 & $\left(5 \cdot 3^{2}\right)$ & $13^{2}$ & 14 & 17 & $(5 \cdot 19)$ & $52^{2}$ \\
2 & 8 & $(5 \cdot 7)$ & $4^{2}$ & 9 & 13 & $(5 \cdot 18)$ & $61^{2}$ & 15 & 17 & $(5 \cdot 23)$ & $100^{2}$ \\
3 & 8 & $\left(5 \cdot 3^{2}\right)$ & $10^{2}$ & 10 & 13 & $(5 \cdot 22)$ & $90^{2}$ & 16 & 17 & $(5 \cdot 29)$ & $261^{2}$ \\
4 & 8 & $(5 \cdot 11)$ & $9^{2}$ & & 13 & $(5 \cdot 22)$ & $90^{2}$ & 17 & 17 & $(5 \cdot 41)$ & $369^{2}$ \\
5 & 8 & $(5 \cdot 31)$ & $81^{2}$ & 11 & 13 & $(5 \cdot 43)$ & $205^{2}$ & 18 & 17 & $(5 \cdot 43)$ & $541^{2}$ \\
6 & 8 & $(5 \cdot 53)$ & $241^{2}$ & 12 & 17 & $\left(5 \cdot 3^{2}\right)$ & $25^{2}$ & 19 & 17 & $(5 \cdot 67)$ & $976^{2}$ \\
$\mathbf{7}$ & $\mathbf{1 3}$ & $(\mathbf{5} \cdot \mathbf{2})$ & $\mathbf{1}$ & 13 & 17 & $(5 \cdot 13)$ & $52^{2}$ & 20 & 17 & $(5 \cdot 71)$ & $1476^{2}$ \\
\hline
\end{tabular}


Table $6\left(G(\mathbf{N} / \mathbb{Q}) \simeq C_{4} \times D_{10}\right)$

\begin{tabular}{ccccccccccc}
\hline & $d_{\mathbf{L}}$ & $h_{\mathbf{L}}$ & $\mathrm{R}_{\mathbf{L}} \leq$ & $\mu_{\mathbf{L}} \leq$ & $f_{\mathbf{N}_{8}}$ & $B_{5}(\mathbf{L})$ & $n_{f}$ & $N_{f}$ & $\mathcal{F}_{\mathbf{N}_{10} / \mathbf{L}}$ & $h_{\mathbf{K}}^{-}$ \\
\hline 1 & 40 & 2 & 1.151 & 0.3719 & 5 & $110^{2}$ & 1 & 0 & - & - \\
2 & 65 & 2 & 1.378 & 0.4718 & 5 & $105^{2}$ & 1 & 1 & 95 & $145305^{2}$ \\
3 & 85 & 2 & 0.959 & 0.6116 & 5 & $55^{2}$ & 0 & 0 & - & - \\
\hline
\end{tabular}

5.3. The cases $\mathbf{G} \simeq C_{3} \times D_{8}$ and $\mathbf{G} \simeq C_{5} \times D_{8}$ (Lattice $I I$ ). In these cases we use the following proposition similar to Proposition 14:

Proposition 16. Let $\mathbf{N}$ be a normal $C M$-field of degree $8 p$ with $G(\mathbf{K} / \mathbb{Q})$ $\simeq C_{p} \times D_{8}$. Assume that $h_{\mathbf{N}}^{-}=1$. If a rational prime $q$ is inert in $\mathbf{L} / \mathbb{Q}$, then $q$ does not divide $f_{\mathbf{N}_{2 p} / \mathbf{L}}$, the norm of the conductor $\mathcal{F}_{\mathbf{N}_{2 p} / \mathbf{L}}$ of $\mathbf{N}_{2 p} / \mathbf{L}$.

Proof. If $h_{\mathbf{N}}^{-}=1$, then $h_{\mathbf{N}_{8}}^{-}=1$ and $\mathbf{N}_{8}$ is the narrow Hilbert 2-class field of some real quadratic field $\mathbf{L}$ in Theorem 11(2). If $q$ is ramified in $\mathbf{N}_{2 p} / \mathbf{L}$, then, since $q$ splits completely in $\mathbf{N}_{8} / \mathbf{L}$ and is ramified in $\mathbf{N} / \mathbf{N}^{+}$, we have $p \mid h_{\mathbf{N}}^{-}$by Proposition 3(2).

We obtain Table 7 in the same way as Table 4 . In Table 7 , to provide the reader with an excerpt of our relative class number computations, for each of the 19 dihedral octic CM-fields $\mathbf{N}_{8}$ of relative class number one, we give the value of the relative class number of the $\mathbf{N}$ with $G(\mathbf{N} / \mathbb{Q}) \simeq C_{3} \times D_{8}$ and containing $\mathbf{N}_{8}$ of least $f_{\mathbf{N}_{6} / \mathbf{L}}$. Table 8 provides the same data for the case $G(\mathbf{N} / \mathbb{Q}) \simeq C_{5} \times D_{8}$. According to these results there is no $\mathbf{N}$ of relative class number one with $\mathbf{G} \simeq C_{3} \times D_{8}$ and $\mathbf{G} \simeq C_{5} \times D_{8}$. In Tables 7 and 8 , $\mathcal{P}_{q}$ denotes the prime ideal of $\mathbf{L}$ above a prime $q$ ramified in $\mathbf{L} / \mathbb{Q}$.

Table $7\left(G(\mathbf{N} / \mathbb{Q}) \simeq C_{3} \times D_{8}\right)$

\begin{tabular}{rccccrrrrr}
\hline & $d_{\mathbf{L}}$ & $h_{\mathbf{L}}$ & $\mathrm{R}_{\mathbf{L}} \leq$ & $\mu_{\mathbf{L}} \leq$ & $B_{3}(\mathbf{L})$ & $n_{f}$ & $N_{f}$ & $\mathcal{F}_{\mathbf{N}_{6} / \mathbf{L}}$ & $h_{\mathbf{N}}^{-}$ \\
\hline 1 & 136 & 2 & 1.458 & 0.6285 & 127000 & 45 & 16 & $(9)$ & $4^{2}$ \\
2 & 205 & 2 & 1.051 & 0.8512 & 22300 & 20 & 11 & $(7)$ & $4^{2}$ \\
$\mathbf{3}$ & $\mathbf{2 2 1}$ & 2 & 0.728 & 1.0622 & 6300 & 15 & 7 & $\mathcal{P}_{\mathbf{1 3}}$ & $\mathbf{1}$ \\
4 & 305 & 2 & 1.578 & 0.9137 & 49700 & 33 & 13 & $(7)$ & $7^{2}$ \\
5 & 377 & 2 & 1.266 & 1.1927 & 19300 & 22 & 10 & $(19)$ & $91^{2}$ \\
6 & 545 & 2 & 1.418 & 1.2455 & 15600 & 19 & 7 & $(13)$ & $52^{2}$ \\
7 & 584 & 2 & 0.939 & 1.4452 & 3700 & 9 & 3 & $(13)$ & $64^{2}$ \\
8 & 712 & 2 & 1.210 & 1.4269 & 6300 & 12 & 8 & $(9)$ & $52^{2}$ \\
9 & 745 & 2 & 2.500 & 0.9936 & 56300 & 31 & 14 & $(9)$ & $67^{2}$ \\
10 & 1345 & 6 & 3.004 & 1.0595 & 40000 & 27 & 10 & $(7)$ & $97^{2}$ \\
11 & 1537 & 2 & 2.626 & 1.2130 & 21800 & 20 & 13 & $(7)$ & $52^{2}$ \\
12 & 1864 & 2 & 1.979 & 1.3345 & 6100 & 11 & 7 & $(7)$ & $109^{2}$ \\
13 & 1945 & 2 & 2.657 & 1.3607 & 16100 & 17 & 6 & $(9)$ & $157^{2}$ \\
14 & 2041 & 2 & 3.362 & 1.1322 & 30200 & 31 & 15 & $(7)$ & $172^{2}$ \\
15 & 2248 & 2 & 1.680 & 1.5128 & 2800 & 7 & 6 & $(7)$ & $112^{2}$ \\
16 & 2329 & 2 & 2.926 & 1.3022 & 16500 & 18 & 6 & $(9)$ & $196^{2}$ \\
17 & 2353 & 2 & 2.612 & 1.3896 & 11500 & 20 & 15 & $\mathcal{P}_{13}$ & $52^{2}$ \\
18 & 4369 & 2 & 3.573 & 1.3589 & 11500 & 15 & 8 & $(7)$ & $217^{2}$ \\
19 & 7081 & 2 & 3.737 & 1.4961 & 6400 & 16 & 11 & $(7)$ & $724^{2}$ \\
\hline
\end{tabular}


Table $8\left(G(\mathbf{N} / \mathbb{Q}) \simeq C_{5} \times D_{8}\right)$

\begin{tabular}{rrrccr}
\hline$d_{\mathbf{L}}$ & $B_{5}(\mathbf{L})$ & $n_{f}$ & $N_{f}$ & $\mathcal{F}_{\mathbf{N}_{10} / \mathbf{L}}$ & \multicolumn{1}{c}{$h_{\mathbf{N}}^{-}$} \\
\hline 136 & 9500 & 6 & 3 & $(11)$ & $71^{2}$ \\
205 & 2400 & 4 & 3 & $\mathcal{P}_{41}$ & $11^{2}$ \\
221 & 900 & 2 & 2 & $(11)$ & $181^{2}$ \\
305 & 4500 & 5 & 3 & $\mathcal{P}_{61}$ & $121^{2}$ \\
377 & 2200 & 4 & 3 & $(11)$ & $1361^{2}$ \\
545 & 1800 & 4 & 2 & $\mathcal{P}_{5}^{3}$ & $1991^{2}$ \\
584 & 600 & 1 & 1 & $(11)$ & $3001^{2}$ \\
712 & 900 & 2 & 0 & $(61)$ & $4250131^{2}$ \\
745 & 5000 & 5 & 3 & $\mathcal{P}_{5}^{3}$ & $4366^{2}$ \\
1345 & 3800 & 5 & 4 & $(11)$ & $18481^{2}$ \\
1537 & 2400 & 4 & 2 & $(31)$ & $2620621^{2}$ \\
1864 & 900 & 2 & 2 & $(11)$ & $26081^{2}$ \\
1945 & 1900 & 4 & 3 & $(11)$ & $39581^{2}$ \\
2041 & 3000 & 4 & 2 & $(25)$ & $1522576^{2}$ \\
2248 & 500 & 2 & 2 & $(11)$ & $83171^{2}$ \\
2329 & 1900 & 4 & 3 & $(25)$ & $2573371^{2}$ \\
2353 & 1400 & 4 & 2 & $\mathcal{P}_{181}$ & $122305^{2}$ \\
4369 & 1400 & 3 & 1 & $(25)$ & $8527696^{2}$ \\
7081 & 900 & 2 & 1 & $(25)$ & $29035651^{2}$ \\
\hline & & & & &
\end{tabular}

5.4. The cases $\mathbf{G} \simeq C_{3} \rtimes D_{8}$ and $\mathbf{G} \simeq C_{5} \rtimes D_{8}$ (Lattice $I$ ). Assume that

$$
G(\mathbf{N} / \mathbb{Q}) \simeq C_{3} \rtimes D_{8} \text { and } h_{\mathbf{N}}^{-}=1 .
$$

For each of the 38 non-normal quartic CM-fields $\mathbf{M}_{1}$ of relative class number one we have computed an upper bound $B_{3}(\mathbf{L})$ such that $f_{\mathbf{N}_{1}^{+} / \mathbf{L}}>B_{3}(\mathbf{L})$ implies $h_{\mathbf{N}_{1}}^{-}>1$. For each possible $\mathbf{N}_{1}$, we have computed $h_{\mathbf{N}_{1}}^{-} / h_{\mathbf{M}_{1}}^{-}$for the non-normal CM-field $\mathbf{N}_{1}$ which is cyclic of degree 3 over a non-normal CM-field $\mathbf{M}_{1}$ by using (2). Finally, our computation shows that in all the cases considered we have $h_{\mathbf{N}_{1}}^{-}>1$, which implies $h_{\mathbf{N}}^{-}>1$. In Table 9, we also give the value of $h_{\mathbf{N}}^{-}$for least $f_{\mathbf{N}_{1}^{+} / \mathbf{L}}$ and we let $n_{f}$ denote the number of conductors of $\mathbf{N} / \mathbf{L}$ satisfying $f_{\mathbf{N}_{1}^{+} / \mathbf{L}} \leq B_{3}(\mathbf{L})$.

For the case $G(\mathbf{N} / \mathbb{Q}) \simeq C_{5} \rtimes D_{8}$ we obtain Table 10 in the same way. In Table 10, we give all possible 12 non-normal CM-fields $\mathbf{N}$ with $\mathbf{G} \simeq C_{5} \rtimes D_{8}$ and $f_{\mathbf{N}_{1}^{+} / \mathbf{L}} \leq B_{5}(\mathbf{L})$.

According to these results there is no $\mathbf{N}$ of relative class number one with $\mathbf{G} \simeq C_{3} \rtimes D_{8}$ or $\mathbf{G} \simeq C_{5} \rtimes D_{8}$. In Tables 9 and 10, $\mathcal{P}_{q}$ denotes a prime ideal of $\mathbf{L}$ above a split prime $q$. Note that there are two possible prime ideals $\mathcal{P}_{q}$. If we choose the other, then we get exactly the other isomorphic non-normal CM-fields $\mathbf{N}_{2}$. 
Table $9\left(G(\mathbf{N} / \mathbb{Q}) \simeq V_{24}=C_{3} \rtimes D_{8}\right)$

\begin{tabular}{|c|c|c|c|c|c|c|}
\hline & $d_{\mathbf{L}}$ & $f_{\mathbf{M}_{1} / \mathbf{L}}$ & $B_{3}(\mathbf{L})$ & $n_{f}$ & $\mathcal{F}_{\mathbf{N}_{1} / \mathbf{L}}$ & $h_{\mathbf{N}}^{-}=\left(h_{\mathbf{N}_{1}}^{-}\right)^{2}$ \\
\hline 1 & 8 & 17 & $120^{2}$ & 10 & $(29) \mathcal{P}_{17}$ & $208^{2}$ \\
\hline 2 & 8 & 73 & $40^{2}$ & 2 & $(29) \mathcal{P}_{73}$ & $148^{2}$ \\
\hline 3 & 8 & 89 & $30^{2}$ & 1 & $(29) \mathcal{P}_{89}$ & $124^{2}$ \\
\hline 4 & 8 & 233 & $20^{2}$ & 0 & - & - \\
\hline 5 & 8 & 281 & $20^{2}$ & 0 & - & - \\
\hline 6 & 5 & 41 & $50^{2}$ & 5 & $(18) \mathcal{P}_{41}$ & $57^{2}$ \\
\hline 7 & 5 & 61 & $40^{2}$ & 3 & $(18) \mathcal{P}_{61}$ & $84^{2}$ \\
\hline 8 & 5 & 109 & $30^{2}$ & 1 & $(18) \mathcal{P}_{109}$ & $63^{2}$ \\
\hline 9 & 5 & 149 & $20^{2}$ & 1 & $(18) \mathcal{P}_{149}$ & $100^{2}$ \\
\hline 10 & 5 & 269 & $20^{2}$ & 1 & $(18) \mathcal{P}_{269}$ & $211^{2}$ \\
\hline 11 & 5 & 389 & $10^{2}$ & 0 & - & - \\
\hline 12 & 13 & 17 & $90^{2}$ & 8 & $(10) \mathcal{P}_{17}$ & $12^{2}$ \\
\hline 13 & 13 & 29 & $60^{2}$ & 5 & $(10) \mathcal{P}_{29}$ & $27^{2}$ \\
\hline 14 & 13 & 157 & $20^{2}$ & 3 & $(10) \mathcal{P}_{157}$ & $196^{2}$ \\
\hline 15 & 13 & 181 & $20^{2}$ & 3 & $(10) \mathcal{P}_{181}$ & $228^{2}$ \\
\hline 16 & 17 & 137 & $30^{2}$ & 1 & $(11) \mathcal{P}_{137}$ & $324^{2}$ \\
\hline 17 & 17 & 257 & $20^{2}$ & 1 & $(11) \mathcal{P}_{257}$ & $444^{2}$ \\
\hline 18 & 29 & 53 & $20^{2}$ & 2 & $(9) \mathcal{P}_{53}$ & $52^{2}$ \\
\hline 19 & 73 & 97 & $30^{2}$ & 1 & ${ }^{(5)} \mathcal{P}_{97}$ & $292^{2}$ \\
\hline 20 & 17 & 8 & $670^{2}$ & 47 & $(11) \mathcal{P}_{2}^{3}$ & $4^{2}$ \\
\hline 21 & 73 & 8 & $550^{2}$ & 35 & $(5) \mathcal{P}_{2}^{3}$ & $16^{2}$ \\
\hline 22 & 89 & 8 & $330^{2}$ & 5 & $(29) \mathcal{P}_{2}^{3}$ & $400^{2}$ \\
\hline 23 & 233 & 8 & $150^{2}$ & 14 & $(17) \mathcal{P}_{2}^{3}$ & $516^{2}$ \\
\hline 24 & 281 & 8 & $190^{2}$ & 7 & $(9) \mathcal{P}_{2}^{3}$ & $208^{2}$ \\
\hline 25 & 41 & 5 & $860^{2}$ & 43 & $(17) \mathcal{P}_{5}$ & $19^{2}$ \\
\hline 26 & 61 & 5 & $360^{2}$ & 34 & $(22) \mathcal{P}_{5}$ & $57^{2}$ \\
\hline 27 & 109 & 5 & $280^{2}$ & 27 & $(11) \mathcal{P}_{5}$ & $36^{2}$ \\
\hline 28 & 149 & 5 & $110^{2}$ & 16 & $(18) \mathcal{P}_{5}$ & $133^{2}$ \\
\hline 29 & 269 & 5 & $70^{2}$ & 3 & $(2) \mathcal{P}_{5}$ & $4^{2}$ \\
\hline 30 & 389 & 5 & $70^{2}$ & 4 & $(2) \mathcal{P}_{5}$ & $4^{2}$ \\
\hline 31 & 17 & 13 & $190^{2}$ & 13 & $(11) \mathcal{P}_{13}$ & $16^{2}$ \\
\hline 32 & 29 & 13 & $60^{2}$ & 6 & $(9) \mathcal{P}_{13}$ & $12^{2}$ \\
\hline 33 & 157 & 13 & $30^{2}$ & 4 & $(10) \mathcal{P}_{13}$ & $43^{2}$ \\
\hline 34 & 181 & 13 & $30^{2}$ & 3 & $(17) \mathcal{P}_{13}$ & $516^{2}$ \\
\hline 35 & 137 & 17 & $50^{2}$ & 3 & $(9) \mathcal{P}_{17}$ & $268^{2}$ \\
\hline 36 & 257 & 17 & $30^{2}$ & 1 & $\mathcal{P}_{17}$ & $4^{2}$ \\
\hline 37 & 53 & 29 & $20^{2}$ & 3 & $(10) \mathcal{P}_{29}$ & $84^{2}$ \\
\hline 38 & 97 & 73 & $30^{2}$ & 2 & $(23) \mathcal{P}_{73}$ & $756^{2}$ \\
\hline
\end{tabular}


Table $10\left(G(\mathbf{N} / \mathbb{Q}) \simeq V_{40}=C_{5} \rtimes D_{8}\right)$

\begin{tabular}{cccc|rrcr}
\hline & $d_{\mathbf{L}}$ & $\mathcal{F}_{\mathbf{N}_{1} / \mathbf{L}}$ & $h_{\mathbf{N}}^{-}=\left(h_{\mathbf{N}_{1}}^{-}\right)^{2}$ & & $d_{\mathbf{L}}$ & $\mathcal{F}_{\mathbf{N}_{1} / \mathbf{L}}$ & $h_{\mathbf{N}}^{-}=\left(h_{\mathbf{N}_{1}}^{-}\right)^{2}$ \\
\hline 1 & 17 & $(79) \cdot \mathcal{P}_{2}^{3}$ & $73205^{2}$ & 7 & 89 & $\left(5^{2}\right) \cdot \mathcal{P}_{2}^{3}$ & $86525^{2}$ \\
2 & 41 & $(109) \cdot \mathcal{P}_{5}$ & $3688955^{2}$ & 8 & 89 & $(59) \cdot \mathcal{P}_{2}^{3}$ & $2732816^{2}$ \\
3 & 41 & $(179) \cdot \mathcal{P}_{5}$ & $5263280^{2}$ & 9 & 109 & $(79) \cdot \mathcal{P}_{5}$ & $2044655^{2}$ \\
4 & 41 & $(199) \cdot \mathcal{P}_{5}$ & $9782005^{2}$ & 10 & 181 & $(19) \cdot \mathcal{P}_{13}$ & $194011^{2}$ \\
5 & 61 & $(59) \cdot \mathcal{P}_{5}$ & $101680^{2}$ & 11 & 257 & $(19) \cdot \mathcal{P}_{17}$ & $1030480^{2}$ \\
6 & 73 & $\left(5^{2}\right) \cdot \mathcal{P}_{2}^{3}$ & $9136^{2}$ & 12 & 389 & $(29) \cdot \mathcal{P}_{5}$ & $228005^{2}$ \\
\hline
\end{tabular}

6. The case $\mathbf{G} \simeq C_{2} \times F_{5,4}$. Let $\mathbf{N}$ be a normal CM-field of degree 40 with Galois group $\mathbf{G}=G(\mathbf{N} / \mathbb{Q}) \simeq C_{2} \times F_{5,4}=C_{2} \times\left\langle a, b: a^{5}=\right.$ $\left.b^{4}=1, b^{-1} a b=a^{2}\right\rangle \simeq\left\langle\sigma, \tau: \sigma^{10}=\tau^{4}=1, \tau^{-1} \sigma \tau=\sigma^{7}\right\rangle$. Note that $\mathbf{N}^{+}$is a normal real field with Galois group $G\left(\mathbf{N}^{+} / \mathbb{Q}\right) \simeq F_{5,4}$. Moreover, $D(\mathbf{G})=\left\langle\sigma^{2}\right\rangle$ and $Z(\mathbf{G})=\left\langle\sigma^{5}\right\rangle$. Hence, $\sigma^{5}$ is the complex conjugation in G. Let $\mathbf{N}_{8}$ be the fixed subfield of the 5-Sylow normal subgroup $D(\mathbf{G})$ of G. Then $\mathbf{N}_{8}$ is an imaginary abelian octic field whose maximal totally real subfield $\mathbf{N}_{8}^{+}$is cyclic quartic, and we let $\mathbf{L}$ denote the quadratic subfield of $\mathbf{N}_{8}^{+}$and $\mathbf{L}_{\mathrm{im}}$ be any one of the two imaginary quadratic subfield of $\mathbf{N}_{8}$. Notice that $w_{\mathbf{N}}=w_{\mathbf{N}_{8}}$. We have the following lattice of subfields:

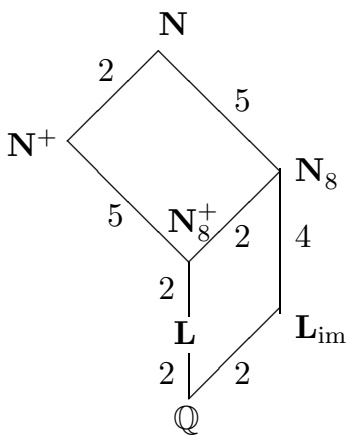

$$
\begin{aligned}
& G\left(\mathbf{N}^{+} / \mathbb{Q}\right) \simeq F_{5,4} \\
& G\left(\mathbf{N}_{8} / \mathbb{Q}\right) \simeq C_{4} \times C_{2} \\
& G\left(\mathbf{N}_{8}^{+} / \mathbb{Q}\right) \simeq C_{4} \\
& G\left(\mathbf{N} / \mathbf{N}_{8}^{+}\right) \simeq C_{10}
\end{aligned}
$$

Proposition 17. Let $\mathbf{K} / \mathbf{M}$ be a cyclic quintic extension of a real cyclic quartic field $\mathbf{M}$. Let $\chi$ be a character of order 5 associated with $\mathbf{K} / \mathbf{M}$. Fix a generator $b$ of $G(\mathbf{M} / \mathbb{Q})$.

(1) $\mathbf{K}$ is a normal number field with Galois group $G(\mathbf{K} / \mathbb{Q}) \simeq F_{5,4}$ if and only if $\mathcal{F}_{\mathbf{K} / \mathbf{M}}$ is invariant under the action of $G(\mathbf{M} / \mathbb{Q})$ and for some $u \in\{2,3\}$ we have $\chi(b(\mathcal{P}))=\chi(\mathcal{P})^{u}$ for all prime ideals $\mathcal{P}$ of $\mathbf{M}$.

(2) Let $\mathbf{K}$ be a normal real field of degree 20 with $G(\mathbf{K} / \mathbb{Q}) \simeq F_{5,4}$. Let $\mathcal{P}_{q}, e_{q}$ and $f_{q}$ denote a prime ideal of $\mathbf{M}$ above a rational prime $q$, its ramification index, and its inertial degree, respectively.

(a) If $q$ does not split completely in $\mathbf{M} / \mathbb{Q}$, then $\mathcal{P}_{q}$ is not inert in $\mathbf{K} / \mathbf{M}$. Moreover, if $\mathcal{P}_{q}$ is ramified in both $\mathbf{M} / \mathbb{Q}$ and $\mathbf{K} / \mathbf{M}$ then $q=5$. 
(b) Let $\mathcal{I}_{5}$ denote the ideal of $\mathbf{M}$ such that $(5)=\mathcal{I}_{5}^{e_{5}}$. Then

$$
\mathcal{F}_{\mathbf{K} / \mathbf{M}}=\mathcal{I}_{5}^{e}\left(\prod q\right)
$$

where $\prod q$ is a finite product of distinct rational primes $q$ 's such that

$$
\begin{cases}q \equiv 1(\bmod 5) & \text { if } f_{q}=1, \\ q \equiv \pm 1(\bmod 5) & \text { if } f_{q}=2, \\ q \neq 1(\bmod p) & \text { if } f_{q}=4,\end{cases}
$$

and either $e=0$ or

$$
\begin{cases}e=2 & \text { if } e_{5}=1, \\ e \in\{2,3\} & \text { if } e_{5}=2, \\ e \in\{2,3,4,6\} & \text { if } e_{5}=4 .\end{cases}
$$

Proof. (1) We first prove the necessity. Let $\Phi_{\mathbf{K} / \mathbf{M}}$ denote the Artin map associated with $\mathbf{K} / \mathbf{M}$. Note that

$$
\chi(b(\mathcal{P}))=\chi(\mathcal{P})^{u} \Leftrightarrow \Phi_{\mathbf{K} / \mathbf{M}}(b(\mathcal{P}))=b^{-1} \Phi_{\mathbf{K} / \mathbf{M}}(\mathcal{P}) b=\Phi_{\mathbf{K} / \mathbf{M}}(\mathcal{P})^{u} .
$$

This shows that if $\mathcal{F}_{\mathbf{K} / \mathbf{M}}$ is invariant under $b$, so is the kernel $\operatorname{Ker}\left(\Phi_{\mathbf{K} / \mathbf{M}}\right)$, which yields the normality of $\mathbf{K}$ (see [Cohn, Thm. 8.2.5]). Therefore, considering the Galois group $G(\mathbf{K} / \mathbb{Q})$, we get the desired result. The sufficiency is easily checked.

(2) First, if $q$ does not split completely in $\mathbf{M} / \mathbb{Q}$ then there exists some $i_{0} \in\{1,2,3\}$ such that $b^{i_{0}}\left(\mathcal{P}_{q}\right)=\mathcal{P}_{q}$. Hence, $\chi\left(b^{i_{0}}\left(\mathcal{P}_{q}\right)\right)=\chi\left(\mathcal{P}_{q}\right)^{u^{i} 0}=\chi\left(\mathcal{P}_{q}\right)$, which gives $\chi\left(\mathcal{P}_{q}\right)=1$, and the first claim of (a) is proved. The last claim of (a) follows from ramification theory.

Second, assume that $q \neq 5$ and $\mathcal{P}_{q}$ divides $\mathcal{F}_{\mathbf{K} / \mathbf{M}}$. Then, since $\mathcal{F}_{\mathbf{K} / \mathbf{M}}$ is invariant under action of $G(\mathbf{M} / \mathbb{Q}),(q)$ divides $\mathcal{F}_{\mathbf{K} / \mathbf{M}}$. By the method of [LPL, Lemma 5] we get $\nu_{q}\left(\mathcal{F}_{\mathbf{K} / \mathbf{M}}\right)=1$, where $\nu_{q}$ denotes the $q$-adic valuation. Note that there exists a primitive modular character of order 5 on $\left(A_{\mathbf{M}} /(q)\right)^{*}$ which is trivial on $\operatorname{Im} \mathbb{Z}$, the image of $\mathbb{Z}$. Hence, the order of $\left(A_{\mathbf{M}} /(q)\right)^{*} / \operatorname{Im} \mathbb{Z}$ must be divisible by 5 .

Third, assume that 5 is ramified in $\mathbf{K} / \mathbf{M}$. It is easily checked that $e>1$. Assume that $e \geq 3$ for $e_{5}=1, e \geq 4$ for $e_{5}=2$, and $e \geq 7$ for $e_{5}=4$. Let $\alpha \equiv 1\left(\bmod \mathcal{P}_{5}^{e-1}\right)$. Then there exists $\beta \in \mathcal{P}_{5}^{e-1-e_{5}}$ such that $\alpha=1+5 \beta$. By using $\nu_{5}\left(C_{k}^{5}\right)=1-\nu_{5}(k)$ for $1 \leq k \leq 5$, we obtain $\alpha \equiv(1+\beta)^{5}\left(\bmod \mathcal{P}_{5}^{e}\right)$, which contradicts the existence of a primitive modular character of order 5 on $\left(A_{\mathbf{M}} / \mathcal{P}_{5}^{e}\right)^{*}$. Finally, by the same trick of [LPL, Lemma 5] we get $e \neq 5$ for $e_{5}=4$, which complete the proof of (b).

THEOREM 18. Let $\chi$ denote any primitive character of order 10 associated with $\mathbf{N} / \mathbf{N}_{8}^{+}$and let $W_{\chi}$ denote the Artin root number associated with $\chi$. Then $W_{\chi}= \pm 1$ and $L(0, \chi) \in 16 \mathbb{Z}$. Moreover, $h_{\mathbf{N}_{8}}^{-}$divides $h_{\mathbf{N}}^{-}$, and 
$h_{\mathbf{N}}^{-} / h_{\mathbf{N}_{8}}^{-}=\left(h_{\mathbf{N} / \mathbf{N}_{8}}^{-}\right)^{4}$ is the 4 th power of the rational integer:

$$
h_{\mathbf{N} / \mathbf{N}_{8}}^{-}=\frac{1}{16} L(0, \chi) \text {. }
$$

Proof. The proof is similar to that of Theorem 5. Let $\sigma_{u}$ denote a generator of the Galois group $G\left(\mathbb{Q}\left(\zeta_{10}\right) / \mathbb{Q}\right)$ such that $\sigma_{u}\left(\zeta_{10}\right)=\zeta_{10}^{u}$, where $\zeta_{10}=e^{2 \pi i / 10}$. Then, since for any ideal $\mathcal{I}, \sigma_{u}(\chi(\mathcal{I}))=\chi(\mathcal{I})^{u}=\chi(b(\mathcal{I}))$ for a generator $b \in G\left(\mathbf{N}_{8}^{+} / \mathbb{Q}\right)$, we conclude that the algebraic number $L(0, \chi)$ which is invariant under the action of $G\left(\mathbb{Q}\left(\zeta_{10}\right) / \mathbb{Q}\right)$ is rational.

To compute numerical approximations of $L(0, \chi)$ by using the technique developed in [Lou5] and [Lou6], we have to be able to compute the coeffi-

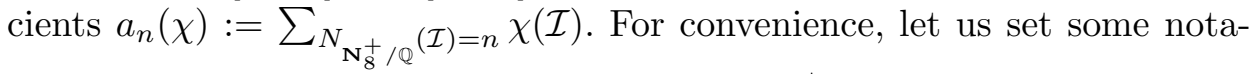
tions. Let $\mathcal{P}_{q}$ and $f_{q}$ denote a prime ideal in $\mathbf{N}_{8}^{+}$above a rational prime $q$ and its inertial degree, respectively. We have:

Proposition 19. Let $\chi_{+}$and $\chi_{-}$denote the characters associated with the cyclic extensions $\mathbf{N}^{+} / \mathbf{N}_{8}^{+}$and $\mathbf{N}_{8} / \mathbf{N}_{8}^{+}$, respectively, such that $\chi=$ $\chi_{+} \chi_{-}$is a character of order 10 associated with $\mathbf{N} / \mathbf{N}_{8}^{+}$. If either $q$ divides $f_{\mathbf{N} / \mathbf{N}_{8}^{+}}$or $f_{q}$ does not divide $k$, then $a_{q^{k}}(\chi)=0$. Otherwise, set $\varepsilon_{q}=\chi_{-}\left(\mathcal{P}_{q}\right)= \pm 1$ and $\eta_{q}=\chi_{+}\left(\mathcal{P}_{q}\right)=\zeta_{5}^{n}$, for some $n \in \mathbb{Z}$. Then

$$
a_{q^{k}}(\chi)= \begin{cases}\varepsilon_{q}^{k} & \text { if } e_{q}=4 \text { and } f_{q}=1, \\ \varepsilon_{q}^{k / 2} & \text { if } e_{q}=2 \text { and } f_{q}=2, \\ \varepsilon_{q}^{k}(k+1) & \text { if } e_{q}=2 \text { and } f_{q}=1, \\ 1 & \text { if } e_{q}=1 \text { and } f_{q}=4, \\ k / 2+1 & \text { if } e_{q}=1 \text { and } f_{q}=2 .\end{cases}
$$

If $e_{q}=f_{q}=1$, then

$$
a_{q^{k}}(\chi)= \begin{cases}\frac{(k+1)(k+2)(k+3)}{6} \varepsilon_{q}^{k} & \text { if } \eta_{q}=1, \\ \varepsilon_{q}^{k} & \text { if } k \equiv 0(\bmod 5) \text { and if } \eta_{q} \neq 1, \\ -\varepsilon_{q}^{k} & \text { if } k \equiv 1(\bmod 5) \text { and if } \eta_{q} \neq 1, \\ 0 & \text { otherwise. }\end{cases}
$$

Proof. Assume that $e_{q}=f_{q}=1$. Then

$$
a_{q^{k}}(\chi)=\varepsilon_{q}^{k} \sum_{\substack{r+s+t+u=k \\ r, s, t, u \geq 0}} \eta_{q}^{r+2 s+3 t+4 u} .
$$

If $\eta_{q} \neq 1$ then since $\sum_{r+s+t+u=k} \eta_{q}^{r+2 s+3 t+4 u}$ is the coefficient of $x^{k}$ in $(1-x) /\left(1-x^{5}\right)=(1-x)\left(\sum_{a \geq 0} x^{5 a}\right)$, we have the desired result. The others are immediate from the definition of $a_{q^{k}}(\chi)$ and ramification theory.

Now, assume that $h_{\mathbf{N}}^{-}=1$. Then $h_{\mathbf{N}_{8}}^{-}=1$, and there are 18 such $\mathbf{N}_{8}$ 's (see $[\mathrm{CK}])$. An easy computation shows that $\zeta_{\mathbf{N}_{8}}(s) \leq 0$ in the range $0<s<1$ 
for these 18 fields $\mathbf{N}_{8}$. Therefore, by using Theorem 10, for each of these 18 fields $\mathbf{N}_{8}$ we can compute an upper bound $B\left(\mathbf{N}_{8}^{+}\right)$such that $h_{\mathbf{N}}^{-}>1$ if $f_{\mathbf{N}^{+} / \mathbf{N}_{8}^{+}}>B\left(\mathbf{N}_{8}^{+}\right)$. In Table 11, we let $f_{\mathbf{N}_{8}^{+}}$and $f_{2}$ denote the conductor of $\mathbf{N}_{8}^{+}$and that of an imaginary quadratic subfield of $\mathbf{N}_{8}$, respectively. In the factorization $f_{\mathbf{N}_{8}^{+}}$, we mark the conductor of a character of order 4 with the bold face. Let $n_{f}$ denote the number of possible conductors of $\mathbf{N}^{+} / \mathbf{N}_{8}^{+}$ satisfying $f_{\mathbf{N}^{+} / \mathbf{N}_{8}^{+}} \leq B\left(\mathbf{N}_{8}^{+}\right)$and let $N_{f}$ denote the number of possible conductors of $\mathbf{N} / \mathbf{N}_{8}^{+}$satisfying $f_{\mathbf{N}^{+} / \mathbf{N}_{8}^{+}} \leq B\left(\mathbf{N}_{8}^{+}\right)$and being filtered by using either Proposition 3(2) or Propositions 3(3) and 17(2)(a). In Table 12, we list the relative class numbers $h_{\mathbf{N}}^{-}=\left(h_{\mathbf{N} / \mathbf{N}_{8}}^{-}\right)^{4}$ of the six CM-fields $\mathbf{K}$ which are obtained in the last column of Table 11. We should point out that in Tables 11 and 12, we used PARI-GP to construct primitive characters of order 5 on the ray class group $C l_{\mathbf{N}_{8}^{+}}\left(\mathcal{F}_{\mathbf{N}^{+} / \mathbf{N}_{8}^{+}}\right)$. According to our computations, the normal CM-field of degree 40 given in Theorem 2 is the only normal CM-field of degree 40 with $G(\mathbf{N} / \mathbb{Q}) \simeq C_{2} \times F_{5,4}$ and relative class number one.

Table $11\left(G(\mathbf{N} / \mathbb{Q}) \simeq C_{2} \times F_{5,4}\right)$

\begin{tabular}{rcrrrrrrrrr}
\hline & $f_{\mathbf{N}_{8}^{+}}$ & $f_{2}$ & $h_{\mathbf{N}_{8}^{+}}$ & $Q_{\mathbf{N}}$ & $w_{\mathbf{N}}$ & $\operatorname{Reg}_{\mathbf{N}_{8}^{+}} \leq$ & $\mu_{\mathbf{N}_{8}^{+}} \leq$ & $B\left(\mathbf{N}_{8}^{+}\right)$ & $n_{f}$ & $N_{f}$ \\
\hline 1 & $\mathbf{5} \cdot 3$ & 3 & 1 & 2 & 30 & 0.2780 & 0.5089 & $23^{4}$ & 2 & 2 \\
2 & $\mathbf{5} \cdot 4$ & 4 & 1 & 2 & 20 & 0.3315 & 0.6025 & $22^{4}$ & 3 & 1 \\
3 & $\mathbf{5} \cdot 7$ & 7 & 1 & 2 & 10 & 0.3441 & 0.9326 & $17^{4}$ & 3 & 2 \\
4 & $\mathbf{5} \cdot 8$ & 8 & 1 & 2 & 10 & 0.4028 & 0.9337 & $17^{4}$ & 2 & 0 \\
5 & $\mathbf{1 3} \cdot 4$ & 4 & 1 & 2 & 4 & 0.3811 & 1.5474 & $12^{4}$ & 0 & 0 \\
6 & $\mathbf{1 3} \cdot 7$ & 7 & 1 & 2 & 2 & 0.3238 & 2.1847 & $9^{4}$ & 0 & 0 \\
7 & $\mathbf{1 6} \cdot 3$ & 3 & 1 & 2 & 6 & 0.6586 & 1.0155 & $19^{4}$ & 0 & 0 \\
8 & $\mathbf{1 6}$ & 4 & 1 & 1 & 4 & 0.4317 & 0.5604 & $31^{4}$ & 1 & 0 \\
9 & $\mathbf{1 6} \cdot 11$ & 11 & 1 & 2 & 2 & 0.4205 & 2.8114 & $9^{4}$ & 0 & 0 \\
10 & $\mathbf{1 6} \cdot 5$ & 20 & 2 & 1 & 2 & 0.6950 & 1.3391 & $17^{4}$ & 2 & 0 \\
11 & $\mathbf{3 7} \cdot 4$ & 4 & 1 & 2 & 4 & 1.4646 & 1.8139 & $10^{4}$ & 0 & 0 \\
12 & $\mathbf{2 9} \cdot 8$ & 8 & 1 & 2 & 2 & 0.7201 & 2.5180 & $6^{4}$ & 0 & 0 \\
13 & $\mathbf{1 6} \cdot 5$ & 4 & 2 & 1 & 4 & 0.6950 & 1.3391 & $15^{4}$ & 2 & 0 \\
14 & $\mathbf{1 6}$ & 3 & 1 & 1 & 6 & 0.4317 & 0.5604 & $15^{4}$ & 0 & 0 \\
15 & $\mathbf{1 6} \cdot 3 \cdot 11$ & 11 & 2 & 1 & 2 & 0.9479 & 2.4740 & $7^{4}$ & 0 & 0 \\
16 & $\mathbf{1 7} \cdot 7 \cdot 3$ & 3 & 2 & 1 & 6 & 3.6084 & 1.9785 & $13^{4}$ & 0 & 0 \\
17 & $\mathbf{1 7} \cdot 7 \cdot 11$ & 11 & 2 & 1 & 2 & 3.7957 & 3.0471 & $7^{4}$ & 0 & 0 \\
18 & $\mathbf{6 1} \cdot 7$ & 7 & 5 & 2 & 2 & 2.2448 & 2.4298 & $6^{4}$ & 2 & 1 \\
\hline
\end{tabular}

Table $12\left(G(\mathbf{N} / \mathbb{Q}) \simeq C_{2} \times F_{5,4}\right.$, all $\left.W_{\mathbf{N}}=1\right)$

\begin{tabular}{ccccc|ccccc}
\hline & $f_{\mathbf{N}_{8}^{+}}$ & $f_{2}$ & $\mathcal{F}_{\mathbf{N}^{+} / \mathbf{N}_{8}^{+}}$ & $h_{\mathbf{N} / \mathbf{N}_{8}}^{-}$ & & $f_{\mathbf{N}_{8}^{+}}$ & $f_{2}$ & $\mathcal{F}_{\mathbf{K}^{+} / \mathbf{N}_{8}^{+}}$ & $h_{\mathbf{N} / \mathbf{N}_{8}}^{-}$ \\
\hline 1 & $5 \cdot 3$ & 3 & $(10)$ & 2 & $\mathbf{4}$ & $\mathbf{5} \cdot \mathbf{7}$ & $\mathbf{7}$ & $(\mathbf{2}) \cdot \mathcal{P}_{\mathbf{5}}^{\mathbf{2}}$ & $\mathbf{1}$ \\
2 & $5 \cdot 3$ & 3 & $(7) \cdot \mathcal{P}_{5}^{2}$ & 5 & 5 & $5 \cdot 7$ & 7 & $(15)$ & 12 \\
3 & $5 \cdot 4$ & 4 & $\mathcal{P}_{5}^{6}$ & 3 & 6 & $61 \cdot 7$ & 7 & $(1)$ & 4 \\
\hline
\end{tabular}


Acknowledgments. The author gratefully appreciates many helpful suggestions by S. Louboutin during the preparation of the paper.

\section{References}

[CK] K.-Y. Chang and S.-H. Kwon, Class numbers of imaginary abelian number fields, Proc. Amer. Math. Soc. 128 (2000), 2517-2528.

[Cohn] H. Cohn, Introduction to the Construction of Class Fields, Cambridge Univ. Press, Cambridge, 1985.

[Lef] Y. Lefeuvre, Corps diédraux à multiplication complexe principaux, Ann. Inst. Fourier (Grenoble) 50 (2000), 67-103.

[LLO] F. Lemmermeyer, S. Louboutin and R. Okazaki, The class number one problem for some non-abelian normal CM-fields of degree 24, J. Théor. Nombres Bordeaux 11 (1999), 387-406.

[Lou1] S. Louboutin, On the class number one problem for non-normal quartic CMfields, Tôhoku Math. J. 46 (1994), 1-12.

[Lou2] -, Corps quadratiques à corps de classes de Hilbert principaux et à multiplication complexe, Acta Arith. 74 (1996), 121-140.

[Lou3] -, Upper bounds on $|L(1, \chi)|$ and applications, Canad. J. Math. 50 (1998), $794-$ 815.

[Lou4] -, The class number one problem for the dihedral and dicyclic CM-fields, Colloq. Math. 80 (1999), 259-265.

[Lou5] -, Computation of relative class numbers of CM-fields by using Hecke L-functions, Math. Comp. 69 (2000), 371-393.

[Lou6] - Computation of $L(0, \chi)$ and of relative class numbers of CM-fields, Nagoya Math. J. 161 (2001), 171-191.

[LO1] S. Louboutin and R. Okazaki, Determination of all non-normal quartic CM-fields and of all non-abelian normal octic CM-fields with class number one, Acta Arith. 67 (1994), 47-62.

[LO2] - - - The class number one problem for some non-abelian normal CM-fields of 2-power degrees, Proc. London Math. Soc. 76 (1998), 523-548.

[LOO] S. Louboutin, R. Okazaki and M. Olivier, The class number one problem for some non-abelian normal CM-fields, Trans. Amer. Math. Soc. 349 (1997), 3657-3678.

[LP] S. Louboutin and Y.-H. Park, Class number problems for dicyclic CM-fields, Publ. Math. Debrecen 57/3-4 (2000), 283-295.

[LPL] S. Louboutin, Y.-H. Park and Y. Lefeuvre, Construction of the real dihedral number fields of degree 2p. Applications, Acta Arith. 89 (1999), 201-215.

[Mar] J. Martinet, Sur l'arithmétique des extensions galoisiennes à groupe de Galois diédral d'ordre 2p, Ann. Inst. Fourier (Grenoble) 19 (1969), no. 1, 1-80.

[Wa] L. C. Washington, Introduction to Cyclotomic Fields, Grad. Texts in Math. 83, Springer, 1997.

\section{CIST}

Korea University

136-701 Seoul, South Korea

E-mail: youngho@semi.korea.ac.kr

Received on 8.9.2000

and in revised form on 17.1.2001 